\title{
Violencia hacia la mujer: características psicológicas y de personalidad de los hombres que maltratan a su pareja
}

\author{
Andrea Torres*, Serafín Lemos-Giráldez y Juan Herrero
}

Departamento de Psicología, Universidad de Oviedo, España

\begin{abstract}
Resumen. Este trabajo presenta una revisión teórica de las principales investigaciones de la última década sobre el estudio de las características psicológicas y de personalidad de los hombres condenados por violencia hacia la mujer en la relación de pareja. La revisión efectuada atiende a tres características principales de los estudios: instrumentos de evaluación, tipo de muestra y tipologías encontradas. En cuanto los instrumentos de evaluación se han utilizado frecuentemente el MCMI en sus tres versiones (Millon Clinical Multiaxial Inventory) STAXI (State-Trait Anger Expression Inventory), SCL-90 (Symptom Checklist-90-R) y PCL-R (Psychopathy Checklist-Revised), entre otros. Respecto a las muestras utilizadas, éstas varían en tamaño y tipo (comunitaria o penitenciaria) en los diferentes estudios. Esta variabilidad tanto en los instrumentos de evaluación como en el tamaño y tipo de las muestras limita claramente la comparación de las características obtenidas. Dentro de la heterogeneidad de los grupos encontrados en las distintas investigaciones existen determinadas características que se repiten de forma recurrente: características del tipo antisocial, narcisista, borderline y abuso de sustancias.

Palabras clave: Violencia en la pareja; maltratadores; tipologías; características psicológicas; trastorno de personalidad.
\end{abstract}

\section{Introducción}

La violencia hacia la mujer en las relaciones de pareja es un problema social de primera magnitud. En nuestro país, se estima que alrededor de un $9.3 \%$ de las mujeres mayores de 18 años son víctimas de maltrato físico, psicológico o sexual por parte de sus parejas y que en más del $60 \%$ de los casos éste ocurre durante un período superior a 5 años (Instituto de la Mujer, 2006; Patró, Corbalán y Limiñana, 2007).

En las últimas décadas se ha producido un aumento de las investigaciones sobre la violencia contra las mujeres por parte de sus parejas debido a su alta prevalencia y a sus consecuencias graves en la salud física, psíquica y emocional a través del tiempo (Alonso, Manso, García-Baamonde Sánchez, 2009; Domínguez, García y Cuberos, 2008). Existen numerosos informes y estudios que muestran las importantes consecuencias que tiene la violencia contra la mujer. Por ejemplo, la investigación realizada por INNOCENTI DIGEST en el año 2000 expone las consecuencias sanitarias más importantes de la violencia contra la mujer en las relaciones de pareja, tanto en lo que refiere a sus efectos sobre la salud física (heridas, fracturas, embarazos no deseados, asma, síndrome de irritación intestinal, etc.) como psicológica (trastornos de ansiedad, disfunciones sexuales, insomnio, baja autoestima, abuso de sustancias o sentimientos de culpa se encuentran entre los efectos psicopatológicos más habituales). Sin obviar, por supuesto, resultados fatales como los suicidios, homicidios o la mortalidad materna.

Según información proporcionada por el Ministerio de

* Dirección para correspondencia [Correspondence address]:

Andrea Torres. Departamento de Psicología, Universidad de Oviedo

(España).E-mail: grupomito@hotmail.com

\begin{abstract}
Title: Violence against woman: Psychological and personality characteristics of men who abuse their female partner.

Abstract. This paper presents a theoretical review of the major findings during the last decade on the study of personality and psychological characteristics of men convicted of violence against their female partner. Three characteristics of the studies are reviewed: assessment tools, sample type and typologies found. The most commonly used assessment tools were the three available versions of MCMI (Millon Clinical Multiaxial Inventory), STAXI (State-Trait Anger Expression Inventory), SCL-90-R (Symptom Checklist-90-R) and PCL-R (Psychopathy Checklist -Revised). Regarding the samples used, they varied in size and type in different studies (e.g. community or penitenciary samples). This variability in both development tools and the size and type of samples clearly limits the comparison of the characteristics found. Within the heterogeneity of the groups found in the different studies, there are certain characteristics that are repeated on a recurring basis: antisocial, narcissistic, borderline characteristics and substance abuse.

Key words: Intimate partner violence; male batterers; typologies; psycho-
\end{abstract} logical characteristics; personality disorders.

Igualdad, durante el 2009, llegaron a los juzgados 135.540 denuncias por violencia contra la mujer por parte de su pareja o ex pareja. La mayor parte de las denuncias procedían de la propia víctima $(72.7 \%)$. El resto de las denuncias procedían fundamentalmente de atestados policiales y partes de lesiones (Ministerio de Sanidad, Política Social e Igualdad, 2010).

Si bien el número de denuncias es un indicador relativamente sencillo de recopilar, presenta problemas de interpretación como indicador directo de la incidencia de la violencia hacia la mujer. Por ejemplo, existe evidencia de que gran parte de los casos de violencia hacia la pareja no se denuncian (ver Gracia y Herrero, 2006). Es por ello que, con el objetivo de acercarse a una verdadera cuantificación del fenómeno, desde el año 1999 el Instituto de la Mujer viene realizando macroencuestas de violencia contra la mujer. En la última macroencuesta realizada en nuestro país, en el año 2006 , se estima que entre el $4 \%$ y el $12.4 \%$ de las mujeres han sido víctimas de este tipo de violencia (Instituto de la Mujer, 2006). En esta macroencuesta, de ámbito nacional, se analiza la violencia contra la mujer diferenciando dos tipos de maltrato, las mujeres que técnicamente se han considerado como maltratadas por responder a determinados criterios de la encuesta es denominado maltrato técnico o "tipo A", y las mujeres que se autoclasifican como maltratadas es denominado maltrato declarado o "tipo B". En las últimas macroencuesta realizadas en España, tomando en cuenta ambos tipos de maltrato, se ha observado un lento descenso registrado en el año 2006 respecto a 1999 (Ministerio de Trabajo y Asuntos Sociales, 2006; Instituto de la Mujer, 2009).

En estos últimos 10 años, entre 2000 y 2010, el total de mujeres fallecidas a causa de la violencia hacia la mujer fue 
de 711, que perdieron la vida a manos de sus parejas o ex parejas (ver Tabla 1); de las cuales, el $40 \%$ de estas mujeres eran de nacionalidad extranjera. Teniendo en cuenta que la población extranjera empadronada hasta ese año era del 9\%, estamos frente a un porcentaje muy elevado de víctimas. Entre las mujeres extranjeras se han incluido las de nacionalidades africanas, latinoamericanas, de los países pertenecientes a la Unión Europea y del resto de Europa.

Tabla 1. Mujeres víctimas mortales de la violencia en la pareja en España (2000-2010) ${ }^{1}$

\begin{tabular}{|c|c|c|c|c|c|c|c|c|c|c|c|}
\hline & 2000 & 2001 & 2002 & 2003 & 2004 & 2005 & 2006 & 2007 & 2008 & $2009 a$ & $2010^{b}$ \\
\hline Españolas & 50 & 36 & 37 & 61 & 53 & 37 & 48 & 43 & 48 & 31 & 44 \\
\hline Extranjeras ${ }^{c}$ & 9 & 9 & 13 & 10 & 17 & 18 & 20 & 28 & 28 & 24 & 29 \\
\hline Desconocida & 4 & 5 & 4 & 0 & 2 & 3 & 0 & 0 & 0 & 0 & 0 \\
\hline Total & 63 & 50 & 54 & 71 & 72 & 57 & 69 & 71 & 76 & 55 & 73 \\
\hline
\end{tabular}

${ }^{1}$ Los datos desde el año 2000 al 2005 proceden del Instituto de la Mujer y a partir del año 2006 proceden de la Delegación de Gobierno para la Violencia de Género, Ministerio de Igualdad.

a En este año hay 4 casos aún en proceso de investigación.

b Datos provisionales hasta el 31 de Diciembre del 2010.

c Incluye mujeres de nacionalidades latinoamericanas, africanas, pertenecientes a la Unión Europea y el resto de Europa.

Considerando la nacionalidad de las víctimas, el número de mujeres extranjeras víctimas de violencia se ha incrementado significativamente. En estos datos se incluyen aquellos casos en los que el agresor es el cónyuge, ex cónyuge, compañero sentimental, ex compañero sentimental, novio o ex novio. Un dato importante a tener en cuenta en estas cifras es que alrededor del $65 \%$ de las víctimas convivían con sus agresores.

En el año 2005 se registró una leve disminución de los casos de mujeres fallecidas por violencia de género, respecto al año anterior, pero en los años posteriores las cifras volvieron a subir incrementando los casos. El mayor aumento se ha registrado en el año 2008, cifra más elevada de los últimos 10 años. En el 2009 hay 4 casos aún en proceso de investigación, los registrados hasta diciembre son 55 , cifra menor que las registradas años anteriores. Sin embargo, en el año 2010 las cifras volvían a aumentar registrando 73 víctimas mortales y un caso en proceso de investigación hasta el 31 de Diciembre.

Desde el año 2006, el Ministerio de Igualdad ha incluido en sus informes estadísticos anuales de violencia hacia la mujer los porcentajes correspondientes a las denuncias y medidas de protección en vigor por parte de la víctima. El porcentaje de las mujeres que habían denunciado a sus agresores se encuentra entre un $20 \%$ al $30 \%$ de los casos aproximadamente, y el porcentaje de mujeres que tenían medidas de protección en vigor es del 10\% al 20\%. En ambos casos, los porcentajes siguen siendo muy bajos como medidas de protección para la víctima.

Todos estos datos, si bien reflejan los problemas para conocer de forma precisa la magnitud del problema, sugieren que la violencia hacia la mujer en las relaciones de pareja es un fenómeno muy extendido en nuestra sociedad que, además, afecta de forma muy específica a las mujeres que son víctima de ella. Es por ello por lo que en las últimas décadas se ha dedicado una especial esfuerzo investigador por comprender cuáles son las posibles causas de este tipo de violencia. Las diferentes perspectivas teóricas consideran que la causa de la violencia hacia la mujer en las relaciones de pareja se sitúa en múltiples ámbitos. Estos niveles o ámbitos harían referencia a: 1) el individuo (desórdenes de personalidad, anomalías psicológicas, conductas adictivas de los maltratadores, etc.) (Dutton y Golant, 1997; FernándezMontalvo y Echeburúa, 2008; Murphy, Taft y Eckhardt, 2007); 2) la familia (graves problemas de comunicación, conflictividad en la relación, relaciones disfuncionales, ausencia de apoyo familiar, aislamiento social de la familia, etc.) (ver Holtzwortz-Munroe y Stuart, 1994, para un análisis; Huang, Zhang y Cao, 2007; Zhao, Zhang, Li, Zhou y Li, 2008); y 3) social (por ejemplo, sociedades machistas, tolerancia social del uso de la violencia, norma de privacidad familiar, actitudes sociales sobre la violencia hacia la mujer en las relaciones de pareja, etc.) (Gracia y Herrero, 2007; Herrero y Gracia, 2005; Strauss, Kaufman y Moore, 1997).

Uno de los principales retos en la investigación de las relaciones de violencia en la pareja ha sido la identificación de las características de los hombres que la ejercen y de los contextos en que se produce con el objetivo de minimizar el riesgo y de poder mejorar las opciones de intervención (Dixon y Browne, 2003). Tomando en cuenta esta perspectiva, el estudio de los perfiles psicológicos de los hombres condenados por violencia contra la mujer se convierte en uno de los elementos fundamentales para implementar programas de intervención eficaces.

\section{El estudio de las tipologías de los hombres violentos}

El estudio de las tipologías de los hombres maltratadores es relativamente reciente. Los altos porcentajes de víctimas de violencia han provocado en la comunidad científica un especial interés por el estudio de las características psicológicas y de personalidad de los hombres que ejercen violencia hacia su pareja (Fernández- Montalvo y Echeburúa, 2005). Es por ello, que el enfoque orientado al estudio de las tipologías ha atraído una gran atención por parte de los investigadores en el área.

A partir de la década de 1980, los investigadores intentaron describir empíricamente las características de los hombres que maltrataban a sus mujeres. Los esfuerzos iniciales 
se centraron en la búsqueda de los elementos comunes que diferenciaban a los hombres maltratadores de aquellos que no lo eran, así como los subgrupos de hombres violentos entre sí (Lohr, Bonge, Witte, Hamberger y LanghinrichsenRohling, 2005). En general, estos estudios indicaban que los hombres violentos suelen presentar carencias psicológicas, déficits cognitivos, desórdenes de personalidad, consumo abusivo de alcohol y drogas, pensamientos distorsionados sobre la inferioridad de la mujer, baja autoestima, dificultad en la comunicación y en la expresión de sus emociones, falta de control de impulsos y una estrecha relación entre la severidad de la violencia y el control de la ira (Dutton y Golant, 1997; Murphy, Taft y Eckhardt, 2007; Echeburúa y Redondo, 2010).

Ferrer, Bosch, García, Manassero y Gili (2004), realizaron un meta-análisis de investigaciones realizadas entre 1988 y 1998 que comparaban a maltratadores y no maltratadores en cuanto a psicopatología y consumo de alcohol y drogas. En este trabajo concluyeron que los hombres que ejercen violencia hacia sus parejas presentan trastornos de personalidad y otro tipo de trastornos psicopatológicos, abusan del alcohol y/o drogas en mayor medida que los hombres no violentos. Las diferencias observadas en cuanto a los trastornos de personalidad hacen referencia a la presencia del trastorno de personalidad esquizotípico y al trastorno depresivo. Esta revisión sugería que los trastornos de personalidad u otra psicopatología, aunque estén presentes en ciertos maltratadores, no constituirían características distintivas de este colectivo. En cuanto al consumo de alcohol y drogas, si bien es mayor entre los hombres violentos, ello no indica que esté vinculado directamente con el maltrato hacia la mujer pero sí que existe cierta relación entre ambos ya que pueden potenciar otros factores de riesgo.

Parte de las clasificaciones tipológicas actuales han replicado empíricamente la propuesta de Holtzworth-Munroe y Stuart (1994) y la propuesta de Gottman et al. (1995). Estos dos grupos de investigadores hace más de 15 años que guían el debate sobre el estudio de las tipologías de los hombres maltratadores (Amor, Echeburúa y Loinaz, 2009).

Sin duda, la publicación de la revisión teórica de Holtzworth-Munroe y Stuart (1994) en el Psychological Bulletin supone uno de los momentos clave para entender la evolución de este campo de estudio. Es en esta revisión de estudios de los últimos 20 años donde se proponen 3 tipos de maltratadores en función de 3 dimensiones: violencia marital, generalmente violentos, y psicopatología/trastorno de personalidad. De acuerdo con los 3 subtipos descritos, encontraron que los hombres violentos sólo en la familia (FO, Family Onb), presentaban baja implicación delictiva, intensidad moderada de la ira, niveles bajos de depresión y de abuso de alcohol. En cambio, el subtipo disfórico/borderline (DB) presentaba baja o moderada implicación delictiva, padecía de un trastorno de personalidad límite o esquizoide, niveles moderados de abuso de alcohol, altos niveles de depresión e ira. El tercer subtipo, generalmente violento/antisocial (GVA), presentaba implicación delictiva, niveles moderados de ira y bajos de depresión, consumo abusivo de alcohol y características de la personalidad antisocial.

Esta revisión sugería que los problemas psicológicos o características de personalidad explicarían en parte la conducta violenta hacia la mujer, fundamentalmente la personalidad antisocial, trastorno narcisista y el trastorno límite. El trastorno de personalidad del tipo antisocial se caracteriza por el desprecio y violación de los derechos de los demás, el tipo narcisista por la grandiosidad, necesidad de admiración y falta de empatía, y por último el tipo límite por presentar cierta inestabilidad en las relaciones interpersonales, en la autoimagen y en la afectividad, así como rasgos de impulsividad.

Unos años más tarde Holtzworth - Munroe y Meehan (2004) encontraron que las tipologías que se habían conceptualizado en 1994 se seguían manteniendo. Sin embargo, al observar la evolución de los sujetos durante 3 años encontraron que la asignación de los maltratadores a las tipologías no era completamente estable. Es por ello que propusieron continuar con el estudio de las 3 dimensiones descritas originalmente (violentos maritales, generalmente violentos, $\mathrm{y}$ psicopatología/trastorno de personalidad).

La clasificación empírica de Gottman et al. (1995), por su parte, se basaba fundamentalmente en la respuesta psicofisiológica que los hombres violentos emiten ante una discusión de pareja. Realizaron una clasificación en un contexto de laboratorio, y propusieron dos tipos de maltratadores en función de su respuesta cardiaca diferencial ante una discusión de pareja. Los maltratadores de tipo I ("cobra") eran aquellos hombres violentos que, ante una discusión de pareja, manifestaban una disminución en su frecuencia cardiaca, exteriorizaban mucha agresividad y desprecio hacia la víctima y se comportaban violentamente con otro tipo de personas (amigos, desconocidos, compañeros de trabajo, etc.). Desde una perspectiva psicopatológica, mostraban características antisociales y agresivo-sádicas, así como una mayor probabilidad de drogodependencia. En este tipo de hombres violentos $(20 \%)$, la conducta agresiva era planificada, expresaban un grado profundo de insatisfacción, no generaban sentimientos de culpa pero eran emocionalmente más abusivos que el grupo de hombres violentos de tipo II. Los maltratadores de tipo II ("pitbull") eran aquellos hombres violentos que presentaban un aumento en su frecuencia cardiaca ante una discusión de pareja. Desde una perspectiva psicopatológica tendían a mostrar trastornos de personalidad por evitación y borderline, características pasivo-agresivas, ira crónica y eran emocionalmente dependientes. En este tipo de hombres violentos la conducta violenta era impulsiva, una conducta mediatizada por la ira que refleja la incapacidad en el control de los impulsos o en la expresión de los afectos.

En España, se realizaron desde los primeros años 90 estudios orientados al análisis de los hombres que ejercen violencia contra la mujer. Las diferentes investigaciones se orientaron a conocer las diversas variables que intervienen en la explicación de la violencia en la pareja. En estos estudios se evaluaron desde la eficacia de los tratamientos psi- 
cológicos basados en la técnicas cognitivo-conductuales, las diferencias en el tipo y gravedad de la conducta violenta hasta conocer las características psicológicas de los hombres violentos. Los dos principales exponentes de investigaciones orientadas al estudio de las características psicológicas son los grupos de Echeburúa (Echeburúa y FernándezMontalvo, 1997), quienes siguiendo con la línea de investigación de Holtzworth- Munroe y Stuart (1994) realizaron diferentes estudios con el objetivo de establecer empíricamente una tipología de agresores contra la pareja en un ámbito español, y de Cáceres (Cáceres, Landeta, Ballús, Gómez, Otero, Rangel y Robles, 1998), quienes realizaron estudios con el fin de replicar los resultados obtenidos por Gottman et al. (1995) con una muestra española.

La línea de investigación de Echeburúa et al. se centró en el perfil diferencial de los agresores en distintos estudios (Echeburúa y Fernández-Montalvo, 2007; FernándezMontalvo y Echeburúa, 2008; Fernández-Montalvo y Echeburúa, 2005) y la aplicación de la aproximación tipológica en entornos penitenciarios y comunitarios (Loinaz y Echeburúa, 2010; Loinaz, Echeburúa y Torrubia, 2010; Loinaz, Ortiz-Tallo, Sánchez y Ferragut, 2011), obteniendo resultados que coinciden parcialmente con las tipologías propuestas en las investigaciones internacionales y estableciendo dos grupos de agresores diferenciados:

1. Los sujetos "violentos con la pareja/estables emocionalmente/integrados socialmente", equivalentes al tipo normalizado, se caracterizan por violencia limitada a la pareja, menor número de distorsiones cognitivas, menor abuso de drogas, menor presencia de antecedentes penales, mayor autoestima, mayor control de la ira, poca presencia de síndromes clínicos y estilos de personalidad compulsivos, narcisistas e histriónicos. Con un estilo de apego predominantemente seguro, tienen mayor empatía y muestran mayor deseabilidad social.

2. Los sujetos "violentos generalizados/poco estables emocionalmente/no integrados socialmente" se caracterizan por violencia que se extiende más allá de la pareja y presentan antecedentes penales con mayor frecuencia. Estos sujetos están más afectados por síntomas clínicos, tienen más distorsiones cognitivas, dependen más del alcohol/drogas y muestran rasgos de personalidad antisocial, paranoide y negativista. Asimismo, son más impulsivos y se muestran menos empáticos que los sujetos del grupo anterior, con estilo de apego inseguro u hostil.

Las investigaciones en este campo aconsejan seguir con el estudio de las principales dimensiones explicativas de los hombres con conducta violenta hacia la mujer, así como explorar sistemas clasificatorios que permitan orientar la intervención. Se propone continuar con el estudio de las 3 dimensiones descritas originalmente: violentos maritales, generalmente violentos y psicopatología/trastorno de personalidad (Holtzworth - Munroe y Meehan, 2004; Lohr et al., 2005; Amor et al., 2009), y evaluar la necesidad de intervenciones más amplias mediante programas de tratamiento para aquellos agresores con diversos problemas psicopatológicos como, por ejemplo, dependencia del alcohol y drogas, trastornos de personalidad, etc. (Murphy et al., 2007).

Una importante contribución del estudio de las tipologías de los agresores es su énfasis en la importancia de los rasgos de personalidad y las características psicopatológicas, tanto para la comprensión de la violencia en la pareja como para el tratamiento de individuos violentos. Por otra parte, se ha sugerido que las tipologías también ayudarán a identificar los diferentes mecanismos etiológicos de la violencia en la pareja (Capaldi y Kim, 2007).

\section{Investigaciones de las características psicoló- gicas y de personalidad de los hombres violen- tos}

Si bien los estudios sobre las tipologías incorporan una amplia variedad de variables en diferentes ámbitos (psicológico, familiar y social), tratando de encontrar los tipos básicos en función de esos ámbitos, existe también una consolidada tradición investigadora que se ha centrado con mayor énfasis en el estudio de las características psicológicas y de personalidad de los hombres violentos contra la mujer en la relación de pareja. Durante los últimos 10 años (ver Tabla 2), la literatura científica en este ámbito ha aportando una gran cantidad de datos derivados de investigaciones con muy diversas muestras e instrumentos de medida que han aportado evidencia empírica sobre las características psicológicas y de personalidad de estos hombres. Los instrumentos de medida más frecuentemente utilizados en estas investigaciones son: el MCMI en sus tres versiones (Millon Clinical Multiaxial Inventory), STAXI (State-Trait Anger Expression Inventory), SCL-90 (Symptom Checklist-90-R), BDI (Beck Depression Inventory) y PCL-R (Psychopathy Checklist-Revised), entre otros.

White y Gondolf (2000) llevaron a cabo un análisis del perfil de 100 hombres condenados judicialmente por maltratar a sus mujeres que habían sido derivados a tratamiento psicológico. Utilizaron el MCMI-III para el estudio de la personalidad de estos sujetos y encontraron seis grupos principales de la personalidad: estilo depresivo/evitador, estilo conformista/narcisista, trastorno límite, trastorno paranoide, trastorno narcisista y trastorno antisocial.

Holtzworth-Munroe, Rehman y Herron (2000) procedieron a revisar las conclusiones del estudio realizado en el año 1994, donde se habían propuesto los 3 subtipos de maltratadores. En este estudio participaron 102 hombres violentos que habían sido físicamente agresivos con sus esposas en el último año y lo compararon con un grupo de 62 hombres no violentos. Llevaron a cabo una serie de análisis de cluster usando medidas de las tres dimensiones descriptivas (es decir, la violencia conyugal del hombre, la violencia generalizada y trastornos de la personalidad), utilizando como instrumento de evaluación el Inventario Clínico Multiaxial de Millon (MCMI-III). De acuerdo con los resultados obtenidos, encontraron cuatro subgrupos: limitado al ámbito familiar, disfórico/borderline, generalmente violento/antisocial y anti- 
social de bajo nivel que se encontraría entre los violentos familiares y el generalmente violento. Este subgrupo tenía puntuaciones moderadas en las medidas de antisocial, violencia conyugal y la violencia en general.

Gondolf y White (2001) en un estudio con 580 hombres violentos que asistían a un programa de intervención para maltratadores, encontraron que casi dos tercios (60\%) de estos hombres presentaban sintomatología subclínica o bajos niveles de disfunción de la personalidad (evaluada a través del MCMI-III). Por ejemplo, aproximadamente la mitad de los perfiles tenían puntuaciones en las medidas del trastorno narcisista y de evitación. En su estudio, los autores indican que los perfiles de estos hombres violentos reflejan una diversidad de niveles y tipos de personalidad y psicopatología.

En el año 2002, Grann y Wedin con una muestra de 88 hombres en prisión condenados por el delito de violencia hacia la pareja, realizaron un estudio basándose en los resultados obtenidos mediante el PCL-R, el HCR-20 (Historial Clinical Risk-20) y el VRAG (Violence Risk Appraisal Guide). De acuerdo con sus resultados, el 51\% de los maltratadores presentaban problemas con el abuso de sustancias, de los cuales el 31\% sólo con el alcohol, el 5\% con otras drogas (incluidas drogas legales e ilegales) y el $16 \%$ con múltiples sustancias. Por otro lado, al analizar los resultados del PCL$\mathrm{R}$ obtuvieron que el $27 \%$ de la muestra presentaban rasgos psicopáticos.

Delsol, Margolin y John (2003), utilizaron el PAI (Personality Assessment Inventory) y otras medidas adicionales de abusos de sustancias en una muestra de 72 hombres violentos que convivían con sus parejas en el momento del estudio. Para el análisis de los datos, utilizaron la técnica de Latent Class Analysis y estudiaron la pertenencia de los sujetos a 3 grupos de maltratadores. El primer grupo, limitados al ámbito familiar, se caracterizaba por niveles bajos de violencia hacia la pareja, bajo nivel de trastornos de personalidad y bajo nivel de abuso de sustancias. El segundo grupo, se caracterizaba por un nivel de violencia medio-alto, generalmente hacia la pareja, con niveles bajos de psicopatología y niveles altos en el abuso de sustancias. Finalmente el tercer grupo, se caracterizaba por niveles altos de distrés psicológico, tales como depresión, personalidades borderline, antisocial y abuso de sustancias.

Belfrage y Rying (2004) realizaron un estudio con 162 hombres en prisión por homicidio contra la pareja que habían tenido lugar en Suecia entre 1990-1999. Para ello, revisaron los informes forenses, aplicaron el PCL-R y realizaron entrevistas para evaluar criterios del DSM-IV (American Psychiatric Association, 1994). Los trastornos de personalidad que encontraron fueron los relacionados con los tipos narcisista $\mathrm{y}$ antisocial.

Lohr, Bonge, Witte, Hamberger y LanghinrichsenRohling (2005) realizaron un estudio sobre la coherencia y precisión de la identificación de las tipologías de los hombres violentos para ordenar los perfiles derivados empíricamente del MCMI en tres grupos: negativista-dependiente, antisocial, y no patológico. Para ello, tuvieron en cuenta 36 perfiles obtenidos a partir del MCMI. Aunque la mayoría de los perfiles se clasificaron con precisión, los evaluadores tenían más dificultad para clasificar algunos de los perfiles "no patológicos": el 40\% se ubicaron en el grupo antisocial y el $6 \%$ en el grupo negativista-dependiente. Estos resultados apoyan la posibilidad de que los hombres involucrados en la violencia doméstica que se consideran patológicos tienen un cierto nivel elevado de conducta antisocial.

En el año 2006, Johnson et al. realizaron un estudio con 230 hombres que fueron condenados judicialmente por un delito de violencia doméstica. El 54\% tenía una condena anterior por el mismo delito. Utilizaron la evaluación psicométrica previo a las estrategias de intervención para investigar los factores implicados en la violencia doméstica: psicopatología y personalidad, control de la ira, estilo de apego, actitudes hacia la violencia doméstica, actitudes machistas, la empatía, autoestima y la deseabilidad social. Utilizaron además un cuestionario, diseñado por el grupo investigador, para recabar información acerca de si habían experimentado violencia en su familia de origen y si había tenido algún abuso en su infancia. Los instrumentos que utilizaron fueron: el MCMI-III, DAS (Love Scale from the Dysfunctional Attitude Scale), RMAS (Acceptance of interpersonal violence and sex role stereotyping from the Rape Myth Acceptance Scale) y SES (Self-Esteem Scale) de Rosenberg. De acuerdo con los resultados obtenidos en el MCMI-III para el estudio de las características de personalidad, encontraron 4 grupos: baja patología, narcisista, borderline y antisocial. El grupo que denominaron de baja patología $(12 \%)$ mostró características de baja dependencia interpersonal, moderadas actitudes machistas y menor ideación suicida. El 13\% puntuaron en la escala narcisista y obtuvieron baja puntuación en las actitudes machistas pero la puntuación de deseabilidad social era alta. El grupo de antisociales $(47 \%)$ fue el más propenso a tener antecedentes penales, tuvieron la mayor tasa de dependencia al alcohol y obtuvieron puntuaciones altas en las actitudes machistas. Por último, el grupo borderline (28\%) obtuvo altas puntuaciones en depresión mayor y dependencia al alcohol, mostraban una mayor incidencia de abuso físico y sexual en la infancia, poseían baja autoestima y los niveles más altos de ira. 
Tabla 2. Investigaciones de los últimos 10 años de las características de personalidad de hombres maltratadores

\begin{tabular}{|c|c|c|c|}
\hline Autores & Muestra & Metodología & Características de Personalidad \\
\hline White y Gondolf (2000) & $\begin{array}{l}100 \text { maltratadores derivados ju- } \\
\text { dicialmente a tratamiento }\end{array}$ & Perfiles del MCMI-III & $\begin{array}{l}\text { Estilo depresivo/evitador } \\
\text { Estilo conformista/narcisista } \\
\text { Trastorno borderline } \\
\text { Trastorno paranoide } \\
\text { Trastorno narcisista } \\
\text { Trastorno antisocial }\end{array}$ \\
\hline $\begin{array}{l}\text { Holtzworth-Munroe et al. } \\
(2000)\end{array}$ & $\begin{array}{l}102 \text { parejas violentas y } 62 \text { pare- } \\
\text { jas no violentas }\end{array}$ & $\begin{array}{l}\text { Análisis de cluster a partir del } \\
\text { MCMI-III y medidas de vio- } \\
\text { lencia }\end{array}$ & $\begin{array}{l}\text { Limitado al ámbito familiar Antisocial de } \\
\text { bajo nivel Disfórico/borderline Generalmen- } \\
\text { te violento/antisocial }\end{array}$ \\
\hline Gondolf y White (2001) & $\begin{array}{l}580 \text { hombres violentos que } \\
\text { asistían a un programa de inter- } \\
\text { vención }\end{array}$ & $\begin{array}{l}\text { Análisis a partir del MCMI- } \\
\text { III }\end{array}$ & $\begin{array}{l}\text { Narcisista } \\
\text { Evitación }\end{array}$ \\
\hline Grann y Wedin (2002) & 88 sujetos en prisión & $\begin{array}{l}\text { Análisis a partir del PCL-R y } \\
\text { otras medidas }\end{array}$ & $\begin{array}{l}\text { Psicopatía } \\
\text { Abuso o dependencia de alcohol o drogas }\end{array}$ \\
\hline $\begin{array}{l}\text { Delsol, Margolin y John } \\
\text { (2003) }\end{array}$ & 72 sujetos & $\begin{array}{l}\text { Análisis a partir del PAI y } \\
\text { medidas de abuso de sustan- } \\
\text { cias }\end{array}$ & $\begin{array}{l}\text { Limitado al ámbito familiar } \\
\text { Abuso de sustancias } \\
\text { Generalmente violentos }\end{array}$ \\
\hline Belfrage y Rying (2004) & $\begin{array}{l}164 \text { maltratadores en prisión } \\
\text { por homicidio }\end{array}$ & $\begin{array}{l}\text { Análisis a partir del PCL-R y } \\
\text { entrevistas para evaluar cri- } \\
\text { terios del DSM-IV }\end{array}$ & $\begin{array}{l}\text { Tipo antisocial } \\
\text { Tipo narcisista }\end{array}$ \\
\hline Lorh et al. (2005) & $\begin{array}{l}36 \text { perfiles de hombres estudia- } \\
\text { dos }\end{array}$ & Análisis a partir del MCMI & $\begin{array}{l}\text { No patológico } \\
\text { Pasivo-agresivo/dependiente Antisocial }\end{array}$ \\
\hline Johnson et al. (2006) & $\begin{array}{l}230 \text { agresores derivados judi- } \\
\text { cialmente a tratamiento }\end{array}$ & $\begin{array}{l}\text { Análisis de cluster a partir del } \\
\text { MCMI-III y otras medidas }\end{array}$ & $\begin{array}{l}\text { Normal } \\
\text { Narcisista } \\
\text { Borderline } \\
\text { Antisocial }\end{array}$ \\
\hline $\begin{array}{l}\text { Murphy, Taft y Eckhardt } \\
(2007)\end{array}$ & 139 hombres violentos & $\begin{array}{l}\text { Análisis de cluster a partir del } \\
\text { STAXI, SRP-II, IIP, CTS, } \\
\text { MMEA y SES }\end{array}$ & $\begin{array}{l}\text { Ira patológica } \\
\text { Bajo control de la ira } \\
\text { Ira normal }\end{array}$ \\
\hline Echeburúa et al. (2008) & 76 sujetos en prisión & $\begin{array}{l}\text { Análisis dimensiones del } \\
\text { MCMI-II y PCL-R }\end{array}$ & $\begin{array}{l}\text { Trastorno obsesivo-compulsivo } \\
\text { Trastorno dependiente } \\
\text { Trastorno paranoide } \\
\text { Trastorno antisocial }\end{array}$ \\
\hline Echeburúa et al. (2009) & $\begin{array}{l}196 \text { sujetos que acudieron vo- } \\
\text { luntariamente a un centro de } \\
\text { atención psicológica }\end{array}$ & $\begin{array}{l}\text { Análisis de dimensiones del } \\
\text { MCMI-II, STAXI, SCL-90, } \\
\text { STAI, EA, BDI }\end{array}$ & $\begin{array}{l}\text { Trastorno compulsivo } \\
\text { Trastorno agresivo } \\
\text { Trastorno narcisista }\end{array}$ \\
\hline $\begin{array}{l}\text { Loinaz, Echeburúa } \\
\text { y Torrubia }(2010)\end{array}$ & $\begin{array}{l}50 \text { sujetos de un centro peni- } \\
\text { tenciario y que acudieron vo- } \\
\text { luntariamente }\end{array}$ & $\begin{array}{l}\text { Análisis de cluster a partir del } \\
\text { MCMC III, STAXI, EA, } \\
\text { IPDMV y CTS-2 }\end{array}$ & $\begin{array}{l}\text { Cluster 1: trastorno compulsivo e histriónico } \\
\text { Cluster 2: Trastorno antisocial y paranoide, } \\
\text { dependencia de alcohol/drogas }\end{array}$ \\
\hline Torres (2010) & $\begin{array}{l}179 \text { sujetos que acudieron a un } \\
\text { programa de intervención }\end{array}$ & $\begin{array}{l}\text { Análisis de cluster a partir del } \\
\text { MCMC II, SCL-90-R y Es- } \\
\text { cala de Impulsividad de } \\
\text { Plutchik }\end{array}$ & $\begin{array}{l}\text { Cluster 1: trastorno antisocial, abuso de dro- } \\
\text { gas e impulsividad } \\
\text { Cluster 2: esquizoide, dependiente, compul- } \\
\text { sivo, esquizotípico, paranoide y trastorno } \\
\text { delirante }\end{array}$ \\
\hline Boira y Jodrá (2010) & $\begin{array}{l}118 \text { hombres que acudieron vo- } \\
\text { luntariamente a un programa de } \\
\text { intervención }\end{array}$ & $\begin{array}{l}\text { Análisis a partir del MCMI- } \\
\text { II y otras medidas relaciona- } \\
\text { das con la presencia de psi- } \\
\text { copatología }\end{array}$ & $\begin{array}{l}\text { Compulsivo, antisocial, agresivo-sádica, nar- } \\
\text { cisista e histriónica } \\
\text { Abuso de drogas/alcohol } \\
\text { Celos patológicos }\end{array}$ \\
\hline $\begin{array}{l}\text { Loinaz, Ortiz-Tallo, } \\
\text { Sánchez y Ferragut (2011) }\end{array}$ & $\begin{array}{l}48 \text { agresores de pareja de un } \\
\text { centro penitenciario }\end{array}$ & $\begin{array}{l}\text { Análisis a partir del MCMI- } \\
\text { III, STAXI-2, IPDMV y } \\
\text { CTS-2. }\end{array}$ & $\begin{array}{l}\text { Grupo } 1 \text { ("normalizados"): narcisista, his- } \\
\text { triónica y compulsivo } \\
\text { Grupo } 2 \text { ("antisociales"): antisocial, para- } \\
\text { noide, trastorno de ansiedad, dependencia } \\
\text { de sustancias y del alcohol, trastorno deli- } \\
\text { rante y bipolar }\end{array}$ \\
\hline
\end{tabular}


Murphy, Taft y Eckhardt, en el año 2007, llevaron a cabo un estudio con 139 participantes que acudían a un programa de investigación clínica sobre hombres violentos contra la pareja. Para ello utilizaron los siguientes instrumentos de evaluación: STAXI, SRP-II (Self-Report Psychopathy Scale-II), IIP (Inventory of Interpersonal Problems), CTS (Conflict Tactics Scales), MMEA (Multidimensional Measure of Emotional Abuse) y SES. A partir del análisis de cluster de las respuestas del STAXI, asignaron a los sujetos a 3 grupos: ira patológica, bajo control de la ira e ira normal.

En el año 2008, Fernández-Montalvo y Echeburúa, con el objeto de conocer con más detalle la presencia de las alteraciones de personalidad (trastorno de personalidad y psicopatía) asociadas a los hombres condenados por violencia grave contra la mujer, realizaron un estudio con 76 hombres que se encontraban en prisión por haber cometido un delito grave de violencia contra la mujer. Para la evaluación utilizaron los siguientes instrumentos: el MCMI-II y el PCL-R. Según los resultados obtenidos en el MCMI-II, el 86.8\% (66 sujetos) de la muestra de agresores presentaban, al menos, un trastorno de personalidad. El trastorno obsesivocompulsivo de la personalidad afectaba al 57.8\% de los casos, el trastorno dependiente de la personalidad se encontró en el $34.2 \%$ de los casos, el trastorno paranoide en el 25\%, y por último, el trastorno antisocial que sólo afectaba al $19.7 \%$.

En el año 2009, Echeburúa et al., evaluaron a 196 sujetos que participaban voluntariamente en un programa terapéutico. De todos los participantes $(\mathrm{N}=196)$, hubo 108 (el 55\%) que lo completaron y 88 (el $45 \%$ ) que lo abandonaron prematuramente. Entre los instrumentos utilizados para el estudio de la personalidad se encontraban: SCL-90-R, STAXI-2, STAI (State-Trait Anxiety Inventory), BDI, MCMI-II y EA (Escala de Autoestima). De acuerdo con los resultados obtenidos, encontraron personas que en muchos casos abusaban del alcohol, que eran inestables emocionalmente (sobre todo, en lo referido al control de la ira y de la ansiedad) y eran frecuentes los trastornos de personalidad, sobre todo el compulsivo, el agresivo y el narcisista.

Loinaz et al. (2010) realizaron un estudio-piloto con el objetivo de establecer empíricamente una tipología de agresores contra la pareja en un ámbito penitenciario español. La muestra constó de 50 sujetos internados en el centro penitenciario Brians-2 (Barcelona) por violencia contra la pareja. Se evaluaron la autoestima, la ira, las distorsiones cognitivas y los trastornos de la personalidad, así como la frecuencia y el tipo de violencia mediante los siguientes instrumentos: EA, STAXI-2, IPDMV (Inventario de Pensamientos Distorsionados), MCMI-III y CTS-2 (Conflict Tactics Scales-2). Respecto a las variables psicopatológicas, la tasa de prevalencia de trastornos de personalidad fue del $38 \%$. En cuanto al abuso de drogas, el 54\% de la muestra presentaba problemas con el alcohol y otras drogas. De acuerdo con los resultados obtenidos para el estudio de la personalidad, encontraron dos grupos, el primero tenía las puntuaciones más altas en todas las escalas de trastornos de la personalidad, respecto al otro grupo, sobre todo en la antisocial y paranoide. En los trastornos dependiente y narcisista no había diferencias significativas entre uno y otro grupo. El otro grupo obtuvo puntuaciones más altas en las escalas compulsiva e histriónica.

Boira y Jordá (2010) realizaron un estudio con 118 hombres que acudieron voluntariamente al programa de intervención para hombres con problemas de control y violencia en el hogar llevado a cabo en el Instituto Aragonés de la Mujer (España). Se evaluaron las características de personalidad de acuerdo con el MCMI-II y otras medidas relacionadas con la presencia de psicopatología, tales como entrevistas semiestructuradas, las cuales recababan datos referidos a la existencia de antecedentes psicológicos o psiquiátricos, el consumo de alcohol y/o drogas y la presencia de comportamiento de celos. El 79.8\% de los hombres a los que se administró el MCMI-II presentaron al menos un trastorno de personalidad: compulsivo, antisocial, agresivo-sádico, narcisista e histriónico.

En ese mismo año, Torres (2010) realizó un estudio con 179 hombres que acudieron a un programa de intervención para maltratadores llevado a cabo en el ámbito comunitario (España) (Lila et al., 2010). Para este estudio se utilizaron como instrumentos de evaluación las entrevistas clínicas, SCL-90-R, MCMI-II y la escala de Impulsividad de Plutchik. De acuerdo con los resultados, se obtuvieron dos grupos claramente definidos y diferenciados por sus características psicológicas y de personalidad. El primer grupo con características del tipo antisocial, relacionado generalmente con el abuso de drogas y con escaso control de las conductas impulsivas, y el segundo grupo con características del tipo esquizoide, dependiente, compulsivo, esquizotípico, paranoide y trastorno delirante, confirmando parcialmente las tipologías propuestas por la literatura científica.

En el último año, Loinaz, Ortiz-Tallo, Sánchez y Ferragut (2011), presentaron un estudio llevado a cabo en un centro penitenciario con 48 agresores de pareja ingresados en la prisión de Alhaurín de la Torre (Málaga) y compararon los resultados con un estudio desarrollado anteriormente con la misma metodología en el centro penitenciario Brians-2 (Barcelona). Para ello utilizaron el MCMI-III, STAXI-2, IPDMV y CTS-2 y obtuvieron dos grupos. El grupo 1 ("rasgos normalizados") se caracterizaba por obtener puntuaciones altas en las escalas narcisista, histriónica y compulsiva, y el grupo 2 ("rasgos antisociales") destacaba por unas puntuaciones significativamente superiores en las escalas de personalidad antisocial, agresivo-sádica, pasivo-agresiva y paranoide. El grupo 2 destacaba en las escalas paranoide y antisocial, y el grupo 1 en compulsiva e histriónica. Si bien, en la muestra de Brians-2 no se daban diferencias en personalidad dependiente $\mathrm{y}$, al igual que en la muestra de Alhaurín de la Torre, los grupos tampoco se diferenciaban en la escala narcisista. A su vez, el grupo 2 presentaba puntuaciones significativamente superiores en todas las escalas clínicas (Eje I), con especial relevancia en trastornos de ansiedad, dependencia de sustancias y del alcohol, trastorno delirante y trastorno bipo- 
lar. Las diferencias se daban en igual medida en ambas muestras penitenciarias.

La revisión de la literatura científica de estos últimos años sobre agresores de pareja pone de manifiesto la alta prevalencia de los trastornos de la personalidad entre los hombres que ejercen violencia hacia su pareja. Entre estos trastornos se han encontrado especialmente el borderline, antisocial, narcisista y paranoide (Echeburúa et al., 2008; Holtzworth-Munroe et al. 2000; Johnson et al., 2006; Loinaz et al. 2011; Lorh et al., 2005; Torres, 2010; White y Gondolf, 2000, entre otros), así como consumo abusivo de alcohol y otras drogas (Boira y Jodrá, 2010; Loinaz, Echeburúa y Torrubia, 2010; Calvete, 2008). Los diferentes resultados obtenidos en estas investigaciones podrían ser útiles y relevantes para el avance en la intervención, para incrementar la eficacia de los programas, para la prevención y educación en el ámbito de la violencia en las relaciones de pareja, ya que las intervenciones dirigidas a los tipos específicos de los hombres maltratadores pueden llegar a ser eficaces para la protección de las mujeres con mayor riesgo de violencia en el futuro. No obstante, estos estudios presentan algunas limitaciones. En primer lugar, una característica específica de las investigaciones llevadas a cabo hasta la fecha es que todas ellas utilizan muestras globales de maltratadores, sin hacer ninguna distinción en cuanto a la gravedad de la violencia ejercida contra las mujeres. En este sentido, la experiencia clínica muestra que no todos los agresores obedecen a un mismo perfil. Probablemente el hombre que ejerce una violencia física extremadamente grave contra su pareja, que incluye en ocasiones intentos frustrados de homicidio, no responde al mismo perfil que, por ejemplo, el maltratador exclusivamente psicológico. En este último caso, sin tratarse en modo alguno de una violencia menor, no pone en peligro la vida de la pareja (Echeburúa et al., 2008). En segundo lugar, la mayoría de estas investigaciones utilizaron autoinformes para sus evaluaciones, por lo que estos resultados pueden estar sesgados por diversos factores tales como la dificultad en la comprensión de los ítems, la deseabilidad social o incluso aparentar padecer una enfermedad mental, entre otros. En este sentido, uno de los instrumentos más utilizado para la evaluación de los trastornos de personalidad en estos estudios (MCMI en sus tres versiones), tiende a sobrediagnosticar los casos clínicos. En tercer lugar, estas investigación no han utilizado un grupo control que permita comparar los resultados obtenidos en esta muestra con hombres no violentos. Aunque se ha reconocido que es difícil obtener un grupo control en este tipo de estudios, esto enriquecería los resultados.

\section{Referencias}

Alonso, M., Manso, J. y García-Baamonde Sánchez, M. (2009). Inteligencia emocional como alternativa para la prevención del maltrato psicológico en la pareja. Anales de Psicología, 25, 250-260.

American Psychiatric Association (1994). Diagnostic and Statistical Manual of mental disorders (4th ed.). Washington, D.C. (trad. castellana: Masson, 1995).

\section{Conclusiones}

El estudio de las características psicológicas y de personalidad de los hombres que ejercen violencia hacia sus mujeres ha tenido un papel relevante en la literatura científica (ver trabajos de Echeburúa y Fernández-Montalvo, 1997; Echeburúa et al., 2009; Gondolf, 1988; Gottman et al., 1995; Holtzworth-Munroe y Stuart, 1994; Loinaz et al. 2011; White y Gondolf, 2000; entre otros).

La comparación de los distintos subtipos de los hombres violentos entre sí, e identificar cómo cada tipo de hombre violento se diferencia de los hombres no violentos, podría aumentar la comprensión de la violencia conyugal y ayudar a identificar los diferentes procesos que subyacen en la violencia en la pareja. El desarrollo de una tipología de los hombres violentos permitiría un examen sistemático de cómo y por qué los hombres utilizan la violencia contra la mujer. (Fernández-Montalvo y Echeburúa, 2008; Holtzworth Munroe y Stuart, 1994; Saunders, 1992, 2002).

Diversos hallazgos en investigaciones previas sugieren que una tipología fiable y válida de los hombres maltratadores pueden aportar información valiosa, con el fin de individualizar los tratamientos psicológicos y llevar a cabo programas preventivos y de intervención adecuados. Existen pruebas de que los hombres que ejercen violencia contra la mujer varían en varios aspectos, que van desde la gravedad de la lesión hasta los rasgos de personalidad. Es por ello que los programas de intervención para maltratadores deben ser diseñados respondiendo a los diferentes aspectos, teniendo en cuenta los niveles de motivación y las diferencias culturales utilizando intervenciones específicas (Saunders, 2002).

Como señalaron algunos investigadores, el diseño de las intervenciones debería realizarse en función de la presencia de trastornos de personalidad o de un problema de abuso de drogas/alcohol, valorando la posibilidad de realizar intervenciones combinadas sobre la violencia y sobre otros problemas complementarios (Jackson, Feder, Forde, Davis, Maxwell y Taylor, 2003; Gondolf, 2009), así como la reincidencia diferencial en función del subtipo de agresor (Loinaz et al. 2011). Por lo tanto, los programas de tratamiento que se centren en las causas de la violencia y en los diferentes contextos tendrán más probabilidades de abordar los principales problemas que subyacen a la violencia y elaborar recomendaciones que permitan alcanzar resultados más positivos (Cavanaugh y Gelles, 2005; Kelly y Johnson, 2008).

Agradecimientos.- Este trabajo ha sido financiado por el programa de Investigación de la Universidad de Oviedo y el Banco Santander (código: UNOV-11-MBSAN-04)

Amor, P., Echeburúa, E., y Loinaz, I. (2009). ¿Se puede establecer una clasificación tipológica de los hombres violentos contra su pareja?. International Journal of Clinical and Health Psychology, 9, 519 - 539.

Belfrage, H. y Rying, M. (2004). Characteristics of spousal homicide perpetrators: a study of all cases of spousal homicide in Sweden 1990-1999. Criminal Behavior and Mental Health, 14, 121-33. 
Boira, S. y Jodrá, P. (2010). Psicopatología, características de la violencia y abandonos en programas para hombres violentos con la pareja: resultados en un dispositivo de intervención. Psicothema, 22, 593-599.

Cáceres, J., Landeta, O., Ballús, E., Gómez, A., Otero, J., Rangel, S. y Robles, O. (1998). Fisiología del desamor: Estar malcasado/a es malo para su salud. C. Medicina Psicosomática, 45/46, 57-69.

Calvete, E. (2008). Características de salud mental de los hombres que maltratan a su pareja. Revista Española de Sanidad Penitenciaria, 10, 49-56.

Capaldi, D. y Kim, H. (2007). Typological approaches to violence in couples: A critique alternative conceptual approach. Clinical Psychology Review, 27, 253-265.

Cavanaugh, M. y Gelles, R. (2005). The Utility of Male Domestic Violence Offender Typologies: New Directions for Research, Policy, and Practice. Journal of Interpersonal Violence, 20, 155-166.

Delsol, C., Margolin, G. y John, R. (2003). A Typology of Maritally Violent Men and Correlates of Violence in Community Sample. Journal of Marriage and Family, 65, 636-651.

Dixon, L. y Browne, K. (2003). The heterogeneity of spouse abuse: A review. Journal of Interpersonal Violence, 21, 1270-1285.

Domínguez, J., García, P. y Cuberos, I. (2008). Violencia contra las mujeres en el ámbito doméstico: consecuencias sobre la salud psicosocial. Anales de Psicología, 24, 115-120.

Dutton, D. y Golant, S. (1997). El golpeador. Un perfil psicológico. Buenos Aires: Paidós.

Echeburúa, E. y Fernández-Montalvo, J. (1997). Tratamiento cognitivoconductual de hombres violentos en el hogar: un estudio piloto. Análisis y Modificación de Conducta, 23, 355-384.

Echeburúa, E. y Fernández-Montalvo, J. (2007). Male batterers with and without psychopathy: An exploratory study in Spanish prisons. International Journal of Offender Therapy and Comparative Criminology, 51, 254-263.

Echeburúa, E. y Redondo, S. (2010). ¿Por qué víctima es femenino y agresor masculino? La violencia contra la pareja y las agresiones sexuales. Madrid: Pirámide.

Echeburúa, E., Fernández-Montalvo, J. y de Corral, P. (2008). ¿Hay una diferencia entre la violencia grave y la violencia menos grave contra la pareja?: un análisis comparativo. International Journal of Clinical and Health Psychology, 8, 355- 382

Echeburúa, E., Sarasua, B., Zubizarreta, I. y de Corral, P. (2009). Evaluación de la eficacia de un tratamiento cognitivo-conductual para hombres violentos contra la pareja en un marco comunitario: una experiencia de 10 años (1997-2007). International Journal of Clinical and Health Psychology, 9, 199-217.

Fernández-Montalvo, J. y Echeburúa, E. (2005). Hombres condenados por violencia grave contra la pareja: Un estudio psicopatológico. Análisis y Modificación de Conducta, 31, 138-475.

Fernández-Montalvo, J. y Echeburúa, E. (2008). Trastornos de personalidad y psicopatía en hombres condenados por violencia grave contra la mujer. Psicothema, 20, 193-198.

Ferrer, V., Bosch, E., García, E., Manassero, M. y Gili, M. (2004). Estudio meta-analítico de características diferenciales entre maltratadores y no maltratadores: el caso de la psicopatología y el consumo de alcohol o drogas. Psykhe, 13, 141-156.

Gondolf, E. (1988). Who are those guys? Toward a behavioral typology of batterers. Violence and Victims, 3, 187-203.

Gondolf, E. (2009). Implementing mental health treatment for batterer program participants: Interagency breakdowns and underlying issues. Violence Against Women, 15, 638-655.

Gondolf, E. y White, R. (2001). Batterer program participants who repeatedly reassault: Psychopathic tendencies and other disorders. Journal of Interpersonal Violence, 16, 361-380.

Gottman, J., Jacobson, N., Rushe, R., Shortt, J., Babcock, J., La Taillade, J. y Waltz, J. (1995). The relationship between heart rate reactivity, emotionally aggressive behavior, and general violence in batterers. Journal of Family Psychology, 9, 227-248.

Gracia, E. y Herrero, J. (2006). Public attitudes Howard reporting partner violence against women and reporting behavior. Journal of Marraige and Family, 68, 759-768.

Gracia, E. y Herrero, J. (2007). Perceived neighborhood social disorder and attitudes toward reporting domestic violence against women. Journal of Interpersonal Violence, 22, 737-752.
Grann, M. y Wedin, I. (2002). Risk factors for recidivism among spousal assault and spousal homicide. Psychology, Crime and Law, 8, 5-23.

Herrero, J. y Gracia, E. (2005). Perceived frequency of domestic violence against women and neighbourhood social disorder. Psychological Reports, 97, 712-716.

Holtzworth-Munroe, A. y Stuart, G. (1994). Typologies of male batterers: Three subtypes and the differences among them. Psychological Bulletin, 116, 476- 497.

Holtzworth - Munroe, A. y Meehan, J. (2004). Typologies of Men Who are Maritally Violent: Scientific and Clinical Implications. Journal of Interpersonal Violence, 19, $1369-1389$

Holtzworth-Munroe, A., Rehman, U. y Herron, K. (2000). General and spouse-specific anger and hostility in subtypes of maritally violent men and nonviolent men. Behavior Therapy, 31, 603-630.

Huang, G., Zhang, Y. y Cao, Y. (2007). Life events, social support and attitude to domestic violence of perpetrators. Chinese Mental Health Journal, 21, 845-848.

Innocenti Research Centre (2000). Domestic Violence Against Women and Girls. Innocenti Digest, 6.

Instituto de la Mujer (2006). La Violencia contra las Mujeres. Resultados de la Macroencuesta. III Parte. Disponible en http://www.mtas.es/mujer/violencia.

Instituto de la Mujer (2009). Las mujeres en cifras 1983-2008. Consultado el 23 de marzo del 2011. Disponible en www.inmujer.migualdad.es/MUJER/publicaciones/docs/Mujeres $\% 20 \mathrm{e}$ n\%20cifras\%201983_2008.pdf

Instituto de la Mujer (2009). Mujeres y hombres en España en el 2009. Consultado el 23 de marzo del 2011. Disponible en http://www.inmujer.migualdad.es/MUJER/mujeres/cifras/index.htm

Jackson, S., Feder, L., Forde, D., Davis, R., Maxwell, C. y Taylor, B. (2003). Batterer Intervention Programs: Where do we go from here? Washington, DC: US Department of Justice.

Johnson, R., Gilchrist, E., Beech, A., Weston, S., Takriti, R. y Freeman, R. (2006). A Psychometric Typology of U.K. Domestic Violence Offenders. Journal of Interpersonal Violence, 21, 1270-1285.

Kelly, J. y Johnson, M. (2008). Differentiation among types of intimate partner violence: research update and implications for interventions. Family Court Review, 46, 476-499.

Lila, M., Catalá, A., Conchell, R., García, A., Lorenzo, M., Pedrón, V. y Terreros, E. (2010). Una experiencia de investigación, formación e intervención con hombres penados por violencia contra la mujer en la Universidad de Valencia: Programa Contexto. Intervención Psicosocial, 19, 167179 .

Lohr, J., Bonge, D., Witte,T., Hamberger, L. y Langhinrichsen-Rohling, J. (2005). Consistency and accuracy of batterer typology identification. Journal of Family Violence, 20, 253-258.

Loinaz, I. y Echeburúa, E. (2010). Necesidades terapéuticas en agresores de pareja según su perfil diferencial. Clínica Contemporánea, 1, 85-95.

Loinaz, I., Echeburúa, E. y Torrubia, R. (2010). Tipología de agresores contra la pareja en prisión. Psicothema, 22, 106-111.

Loinaz, I., Ortiz-Tallo, M., Sánchez, L. y Ferragut, M. (2011). Clasificación multiaxial de agresores de pareja en centros penitenciarios. International Journal of Clinical and Health Psychology, 11, 249-268.

Ministerio de Igualdad (2009). Información estadística de violencia de género. Informes anuales. Consultado el 15 de abril. Disponible en http:/ / www.migualdad.es/ss / Satellite?c=Page\&cid=1193049890202\&l anguage $=$ cas_ES\&pagename $=$ MinisterioIgualdad $\% 2 \mathrm{FPage} \% 2 \mathrm{FMIGU}$ listad oCategorias

Ministerio de Sanidad, Política Social e Igualdad (2010). III Informe anual del Observatorio Estatal de violencia sobre la mujer. Madrid

Ministerio de Trabajo y Asuntos Sociales (2006). III Macroencuesta sobre la violencia contra las mujeres. Informe de resultados. Madrid.

Murphy, C., Taft, C. y Eckhardt, C. (2007). Anger problem profiles among partner violent men: Differences in clinical presentation and treatment outcome. Journal of Counseling Psychology, 54, 189-200.

Patró, R., Corbalán, F. y Limiñana, R. (2007). Depresión en mujeres maltratadas: Relaciones con estilos de personalidad, variables contextuales y de la situación de violencia. Anales de Psicología, 23, 118-124. 
Saunders, D. (1992). A typology of men who batter women: Three types derived from cluster analysis. American Journal Orthopsychiatry, 62, 264-275.

Saunders, D. (2002). Developing guidelines for domestic offender programs: What can we learn from related fields and current research? Journal of Aggression, Maltreatment and Trauma, 5, 235-248.

Strauss, M, Kaufman, G. y Moore, D. (1997). Change in cultural norms approving marital violence from 1968 to 1994. En G. Kaufman y J. Jasinski (Eds.), Out of darkness: Contemporary perspectivas on family violence (pp. 3-16). Thousand Oaks, CA: Sage.
Torres, A. (2010). Violencia en la pareja: perfil psicológico de los hombres violentos. Trabajo de investigación no publicado. Universidad de Oviedo.

White, R. y Gondolf, E. (2000). Implications of personality profiles for batterer treatment. Journal of Interpersonal Violence, 15, 467-488.

Zhao, X., Zhang, Y., Li L., Zhou, Y. y Li, H. (2008). Logistic regression analysis of the psychosociology of physical domestic violence on male perpetrators. Chinese Journal of Clinical Psychology, 16, 210-212.

(Artículo recibido: 24-05-2011, revisión: 28-11-2011, aceptado: 07-12-2011) 